\title{
NOTE ON WEIERSTRASS' $E$-FUNCTION IN THE CALCULUS OF VARIATIONS
}

\author{
By A. E. H. Lore.
}

[Received December 14th, 1907.-Read December 12th, 1907.]

THE object of this Note is not to present rigorous formal proofs of theorems in the Calculus of Variations, but to simplify the customary methods of introducing the $E$-function by substituting for them a more intuitional method.*

'The' $E$-function has been developed chiefly in connection with integrals of the form

$$
\int F\left(x, y, y^{\prime}\right) d x
$$

where $y^{\prime}$ denotes $d y / d x$, and it is understood that the integral is taken along a curve joining two fixed points. For many purposes it is convenient to use Weierstrass' method of parametric representation, in which the coordinates $x, y$ are regarded as functions of a parameter $\theta$; and then the integral takes the form

$$
\int f(x, y, \dot{x}, \dot{y}) d \theta
$$

where $\dot{x}$ and $\dot{y}$ denote $d x / d \theta$ and $d y / d \theta$, and the function $f$ is homogeneous. of the first degree, though not necessarily rational, in $\dot{x}, \dot{y}$. It is known that the integral cannot be a maximum or a minimum unless the curve along which it is taken is one of the curves determined by the differential equation

$$
\frac{d}{d x}\left(\frac{\partial F}{\partial y^{\prime}}\right)-\frac{\partial F}{\partial y}=0
$$

Such curves have been called "stationary" curves or "extremals" of the integral. The question arises-Is the integral taken along a stationary

* Reference may be made to the treatises by A. Kneser, Lehrbuch der Variationsrechuung, Braunschweig, 1900, and O. Bolza, Lectures on the Caliulıs of Variations, Chicago, 1904. 
curve a maximum or a minimum? It is in connection with this question that the $E$-function is introduced.

Denote the fixed end points by $A, B$, the value of the integral $\int F d x$ taken along the stationary curve joining two points $P, Q$ by $(P, Q)$, using a round bracket, the value of the integral taken along any other assigned curve joining the same points by $[P, Q]$, using a square bracket. Let any assigned curve other than the stationary curve $A B$ be drawn joining the fixed end points $A, B$. Then the variation of the integral is expressed by $[A, B]-(A, B)$. If this expression has the same sign for all curves which join the same two points, and lie in a sufficiently small neighbourhood of the stationary curve $A B$, the integral taken along that stationary curve is certainly a maximum or a minimum (a minimum if the sign is + , a maximum if it is $\rightarrow$.

In the figure $A L Q P M B$ represents the varied curve, and $A N B$ the stationary curve joining $A, B$. The expression. $[A, B]-(A, B)$, or the

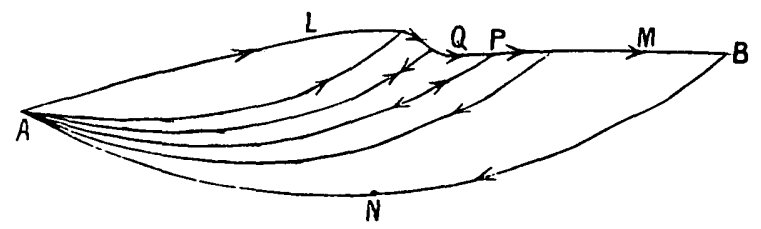

variation of the integral, is equal to the line integral of $F d x$ taken round the closed contour $A L Q P M B N A$. This line integral can be transformed into a line integral taken along the varied curve $A L Q P M B$ only, and can then be written $\int E d s$, where $d s$ denotes the element of arc of the varied curve. The function $E$ is Weierstrass' $E$-function. Since the integral of this function yields the excess of the integral of $F d x$ taken along the varied curve above the integral of $F d x$ taken along the stationary curve, I have elsewhere called it the "excess-function."

The transformation of the line integral taken round the closed contour is effected by the familiar device of breaking up the included region into compartments, and replacing the line integral by a sum of line integrals taken round the boundaries of the compartments, the common boundary of two adjacent compartments being traversed twice in opposite senses, as is indicated by the arrows in the figure. The compartments are bounded by two stationary curves such as $A Q, A P$ joining the point $A$ to points $Q, P$ on the varied curve, and by the intercepted arc $Q P$ of that curve. The line integral of $F d x$ taken round the boundary of one compartment is denoted by $(A, Q)+[Q, P]-(A, P)$, and the variation of the integral is denoted by

$$
\Sigma\{(A, Q)+[Q, P]-(A, P)\},
$$


where the summation extends to all the compartments in a series determined by a series of points, such as $Q, P$, taken in order on the arc of the varied curve. When $Q P$ is made very short the expression $(A, Q)+[Q, P]-(A, P)$ passes over into the product $E d s$, where $d s$ denotes the differential element of arc of the curve $A Q P B$, and $E$ is a certain function, which depends upon the position of $P$, the direction of the tangent at $P$ to the stationary curve $A P$, and the direction of the tangent at $P$ to the varied curve $A Q P B$. The line integral of $F d x$ taken round the contour $A L Q P M B N A$ is therefore transformed into the integral of $E$ taken along the varied curve from $A$ to $B$. It remains to evaluate $E$.

Now the expression $(A, Q)-(A, P)$ may be evaluated simply by means of Lagrange's rule for the variation of an integral in the case of variable limits. Let $\psi$ denote the angle of slope of the varied curve at $P$, in the sense from $Q$ to $P$, and let $\phi$ denote the angle of slope of the stationary curve $A P$ at $P$ in the sense from $A$ to $P$; also let $\delta s$ denote the arc $P Q$, supposed very short. Then Lagrange's rule gives the result

$$
\begin{aligned}
& (A, P)-(A, Q) \\
& \quad=\delta s \cos \psi\left\{F(x, y, \tan \phi)+(\tan \psi-\tan \phi) \frac{\partial F(x, y, p)}{\partial p}\right\}_{p=\tan \phi},
\end{aligned}
$$

which is correct to the first order in $\delta s$. Also we have, correctly to the same order,

$$
[Q, P]=F(x, y, \tan \psi) \delta s \cos \psi,
$$

and therefore, to the same order,

where

$$
(A, Q)+[Q, P]-(A, P)=E \delta s,
$$

$$
\begin{aligned}
E & =\cos \psi \\
& \times\left\{F(x, y, \tan \psi)-F(x, y, \tan \phi)-(\tan \psi-\tan \phi) \frac{\partial F(x, y, p)}{\partial p}\right\}_{p=\tan \phi} .
\end{aligned}
$$

It may be observed parenthetically that Lagrange's rule is proved very easily by the aid of the parametric method. In the integral

$$
\int f(x, y, \dot{x}, \dot{y}) d \theta
$$

let $x$ be changed into $x+\epsilon u$, and $y$ into $y+\epsilon v$, where $\epsilon$ is a constant, and $u$ and $v$ are functions of $\theta$ which, with their first differential coeflicients, are continuous, and are independent of $\epsilon$. The expressions $\epsilon u$, $\epsilon v$ are equivalent to Lagrange's $\delta x, \delta y$. When the first variation of the 
integral is transformed in the usual way by integration by parts, the terms at the limits become

$$
\epsilon\left(u \frac{\partial f}{\partial \dot{x}}+v \frac{\partial f}{\partial \dot{y}}\right),
$$

and the terms under the integral sign vanish, the integral being taken along a stationary curve. If the upper limit is variable in such a way that the corresponding end point $P$ moves on a given curve, the first variation becomes

$$
\delta x\left(\frac{\partial f}{\partial \dot{x}}+\frac{\delta y}{\delta x} \frac{\partial f}{\partial \dot{y}}\right) \text {. }
$$

In this expression $\delta x, \delta y$ are the increments of the coordinates $x, y$ required to pass from the point $P$ to a neighbouring point $Q$ on the given curve, so that, in the notation already employed, we have, with sufficient approximation,

$$
\delta x=-\delta s \cos \psi, \quad \delta y=-\delta s \sin \psi .
$$

Further, since

$$
f=\dot{x} F\left(x, y, \frac{\dot{y}}{\dot{x}}\right),
$$

we have

$$
\frac{\partial f}{\partial \dot{x}}=F-y^{\prime} \frac{\partial F}{\partial y^{\prime}}, \quad \frac{\partial f}{\partial \dot{y}}=\frac{\partial F}{\partial y^{\prime}},
$$

and thus the expression for the first variation of the integral becomes

$$
-\delta s \cos \psi\left\{F\left(x, y, y^{\prime}\right)+\left(\tan \psi-y^{\prime}\right) \frac{\partial F}{\partial y^{\prime}}\right\} .
$$

This first variation expresses, correctly to the first order in $\delta s$, the excess of the value of the integral of $F d x$ taken along the stationary curve $A Q$. above the value of the integral of $F d x$ taken along the stationary curve $A P$, or it is the quantity that has been denoted above by $(A, Q)-(A, P)$.

In terms of the $E$-function we have two important known results in regard to the question of maxima and minima. In the first place, we may take the point $P$ on the stationary curve $A B$ and calculate the $E$. function for the point $P$, the direction $\phi$ of the stationary curve $A B$ at $P$ and an arbitrary direction $\psi$. Let $Q$ be a point near to $P$, so that $Q P$ is in the direction $\psi$, and let the integral of $F d x$ be taken along a varied curve consisting of the arc $A Q$ of the stationary curve $A Q$, the straight line $Q P$, and the arc $P B$ of the stationary curve $A B$, and let this value be compared with that of the integral of $F d x$ taken along the arc $A B$ of the stationary curve $A B$. The variation of the integral is $E \delta s$, where $E$ is the excess-function calculated for the point $P$, the direction $\phi$ of the tangent at $P$ to the stationary curve $A P$, and the direction $\psi$ of the line 
QP. Hence it is a condition for a maximum or minimum that $E$, so calculated, should have the same sign at all points $P$ of $A B$ and for all directions of $Q P$. This condition is necessary. In the second place we have the sufficient condition, that the integral taken along the stationary curve $A B$ is certainly a maximum or a minimum if the function $E$ is onesigned, this function being calculated for all points $P$ in a finite neighbourhood of the stationary curve $A B$, for the direction of the tangent at $P$ to the stationary curve $A P$, and for an arbitrary direction $\psi$ of the tangent at $P$ to a varied curve. In regard to the statement that this condition is sufficient, it is, of course, to be understood that the stationary curve $A B$ or $A P$ must be determined uniquely by its end-points, or that there is not on the arc $A B$ a pair of "conjugate" points. This remark applies to all the arguments in this Note. 